\title{
Article \\ A Non-Standard Finite Difference Scheme for Magneto-Hydro Dynamics Boundary Layer Flows of an Incompressible Fluid Past a Flat Plate
}

\author{
Riccardo Fazio * and Alessandra Jannelli
}

Citation: Fazio, R.; Jannelli, A. A Non-Standard Finite Difference Scheme for Magneto-Hydro Dynamics Boundary Layer Flows of an Incompressible Fluid Past a Flat Plate. Math. Comput. Appl. 2021, 1, 0. https://dx.doi.org/
Department of Mathematics, Computer Science, Physical Sciences and Earth Sciences, University of Messina, Viale F. Stagno D’Alcontres 31, 98166 Messina, Italy; ajannelli@unime.it

* Correspondence: rfazio@unime.it

Abstract: This paper deals with a non-standard finite difference scheme defined on a quasiuniform mesh for approximate solutions of the Magneto-Hydro Dynamics (MHD) boundary layer flow of an incompressible fluid past a flat plate for a wide range of the magnetic parameter. We show how to improve the obtained numerical results via a mesh refinement and a Richardson extrapolation. The obtained numerical results are favourably compared with those available in the literature.

Keywords: MHD model problem; boundary problem on semi-infinite interval; non-standard finite difference scheme; quasi-uniform mesh; error estimation

MSC: 65L10; 65L12; 65L70

\section{Introduction}

The simplest example of the application of the boundary layer theory is related to the celebrated Blasius [1] problem. This problem describes the flow around a very thin flat plate.

The first goal of this paper is to solve numerically, with great accuracy, the MHD boundary layer equation governing the flow of an incompressible fluid past a flat plate by a non-standard finite difference scheme defined on a quasi-uniform mesh. Numerical methods for problems like the one considered in this paper can be classified according to the numerical treatment of the boundary condition imposed at infinity. The oldest and simplest treatment is to replace infinity with a suitable finite value, the so-called truncated boundary. However, being the simplest approach this has revealed within the decades some drawbacks that suggest not to apply it, especially if we have to face a given problem without any clue on its solution behaviour. Several other treatments have been proposed in the literature to overcome the shortcomings of the truncated boundary approach. In this research area they are worthy of consideration: the formulation of socalled asymptotic boundary conditions by de Hoog and Weiss [2], Lentini and Keller [3] and Markowich [4,5]; the reformulation of the given problem in a bounded domain as studied first by de Hoog and Weiss and developed more recently by Kitzhofer et al. [6]; the free boundary formulation proposed by Fazio [7] where the unknown free boundary can be identified with a truncated boundary; the treatment on the original domain via pseudo-spectral collocation methods, see the book by Boyd [8] or the review by Shen and Wang [9] for more details on this topic; and, finally, a non-standard finite difference scheme on a quasi-uniform grid defined on the original domain by Fazio and Jannelli [10]. This non-standard finite difference scheme has been successively modified by Fazio and Jannelli [11]. Moreover, this method has been applied to the numerical solution of the perpetual American put option problem of financial markets, see Fazio [12]. 
This study concludes by comparing the current numerical results with those given by the integral approximation method (ITM) and the non-integral technique (NIT) used by Singh and Chandarki [13].

\section{Model Problem}

We consider a steady two-dimensional flow of a viscous fluid on a flat plate in the presence of a given transverse magnetic field with small electric conductivity and large transverse magnetic field. Introducing appropriate similarity variables, the governing equations can be reduced to the following boundary value problem (BVP) [13]

$$
\begin{gathered}
\frac{d^{3} u}{d x^{3}}+u \frac{d^{2} u}{d x^{2}}+\beta\left(1-\frac{d u}{d x}\right)=0 \\
u(0)=\frac{d u}{d x}(0)=0, \quad \frac{d u}{d x}(\infty)=1,
\end{gathered}
$$

where $\beta$ is the magnetic parameter. Let us notice that for $\beta=0$ the BVP (1) reduces to the celebrated Blasius problem [1]. For a general class of problems, including (1), results on the existence, uniqueness, and boundedness of solutions were obtained by Brighi [14].

\section{The Finite Difference Scheme}

Without loss of generality, we consider the class of BVPs

$$
\begin{aligned}
& \frac{d \mathbf{u}}{d x}=\mathbf{f}(x, \mathbf{u}), \quad x \in[0, \infty), \\
& \mathbf{g}(\mathbf{u}(0), \mathbf{u}(\infty))=\mathbf{0},
\end{aligned}
$$

where $\mathbf{u}(x)$ is a $d$-dimensional vector with ${ }^{\ell} u(x)$ for $\ell=1, \ldots, d$ as components, $\mathbf{f}$ : $[0, \infty) \times \mathbb{R}^{d} \rightarrow \mathbb{R}^{d}$, and $\mathbf{g}: \mathbb{R}^{d} \times \mathbb{R}^{d} \rightarrow \mathbb{R}^{d}$. Here, and in the following, we use Lambert's notation for the vector components [15, pp. 1-5].

In order to solve the problem (2) on the original domain, we discuss first quasiuniform grids maps from a reference finite domain and introduce on the original domain a non-standard finite difference scheme that allows us to impose the given boundary conditions exactly. Let us consider the smooth strict monotone quasi-uniform maps $x=x(\xi)$, the so-called grid generating functions, see Boyd [8, pp. 325-326] or Canuto et al. [16, p. 96],

$$
x=-c \cdot \ln (1-\xi)
$$

and

$$
x=c \frac{\xi}{1-\xi},
$$

where $\xi \in[0,1], x \in[0, \infty]$, and $c>0$ is a control parameter. So that, a family of uniform grids $\xi_{n}=n / N$ defined on interval $[0,1]$ generates one parameter family of quasiuniform grids $x_{n}=x\left(\xi_{n}\right)$ on the interval $[0, \infty]$. The two maps (3) and (4) are referred to as logarithmic and algebraic maps, respectively. As far as the authors' knowledge is concerned, van de Vooren and Dijkstra [17] were the first to use these kinds of maps. We notice that more than half of the intervals are in the domain with length approximately equal to $c$ and $x_{N-1}=c \ln N$ for (3), while $x_{N-1} \approx c N$ for (4). For both maps, the equivalent mesh in $x$ is nonuniform with the most rapid variation occurring with $c \ll x$. The logarithmic map (3) gives slightly better resolution near $x=0$ than the algebraic map (4), while the algebraic map gives much better resolution than the logarithmic map as $x \rightarrow \infty$. In fact, it is easily verified that

$$
-c \cdot \ln (1-\xi)<c \frac{\xi}{1-\xi},
$$


for all $\xi$, but $\xi=0$.

The problem under consideration can be discretized by introducing a uniform grid $\xi_{n}$ of $N+1$ nodes in $[0,1]$ with $\xi_{0}=0$ and $\xi_{n+1}=\xi_{n}+h$ with $h=1 / N$, so that $x_{n}$ is a quasi-uniform grid in $[0, \infty]$. The last interval in (3) and (4), namely $\left[x_{N-1}, x_{N}\right]$, is infinite but the point $x_{N-1 / 2}$ is finite, because the non integer nodes are defined by

$$
x_{n+\alpha}=x\left(\xi=\frac{n+\alpha}{N}\right),
$$

with $n \in\{0,1, \ldots, N-1\}$ and $0<\alpha<1$. These maps allow us to describe the infinite domain by a finite number of intervals. The last node of such grid is placed on infinity so right boundary conditions are taken into account correctly.

We approximate the values of the scalar variable $u(x)$ and its derivative at midpoints of the grid $x_{n+1 / 2}$, for $n=0, \cdots, N-1$, using non-standard difference discretizations

$$
\begin{aligned}
u_{n+1 / 2} & \approx \frac{x_{n+3 / 4}-x_{n+1 / 2}}{x_{n+3 / 4}-x_{n+1 / 4}} u_{n}+\frac{x_{n+1 / 2}-x_{n+1 / 4}}{x_{n+3 / 4}-x_{n+1 / 4}} u_{n+1}, \\
\left.\frac{d u}{d x}\right|_{n+1 / 2} & \approx \frac{u_{n+1}-u_{n}}{2\left(x_{n+3 / 4}-x_{n+1 / 4}\right)} .
\end{aligned}
$$

We emphasize that the key advantage of our non-standard finite difference formulation is to overcome the difficulty of the numerical treatment of the boundary conditions at the infinity. In fact, the formulae (5) use the value $u_{N}=u(\infty)$, but not $x_{N}=\infty$ and then, the boundary conditions at infinity are taken into account in a natural way.

For the class of BVPs (2), a non-standard finite difference scheme on a quasi-uniform grid can be defined by using the approximations given by (5) above, and it can be written as follows

$$
\begin{gathered}
\mathbf{U}_{n+1}-\mathbf{U}_{n}-a_{n+1 / 2} \mathbf{f}\left(x_{n+1 / 2}, b_{n+1 / 2} \mathbf{U}_{n+1}+c_{n+1 / 2} \mathbf{U}_{n}\right)=\mathbf{0}, \\
\text { for } n=0,1, \ldots, N-1 \\
\quad \mathbf{g}\left(\mathbf{U}_{0}, \mathbf{U}_{N}\right)=\mathbf{0},
\end{gathered}
$$

where

$$
\begin{aligned}
& a_{n+1 / 2}=2\left(x_{n+3 / 4}-x_{n+1 / 4}\right), \\
& b_{n+1 / 2}=\frac{x_{n+1 / 2}-x_{n+1 / 4}}{x_{n+3 / 4}-x_{n+1 / 4}}, \\
& c_{n+1 / 2}=\frac{x_{n+3 / 4}-x_{n+1 / 2}}{x_{n+3 / 4}-x_{n+1 / 4}},
\end{aligned}
$$

for $n=0,1, \ldots, N-1$. The finite difference formulation (6) has order of accuracy $O\left(N^{-2}\right)$. It is evident that (6) is a nonlinear system of $d(N+1)$ equations in the $d(N+1)$ unknowns $\mathbf{U}=\left(\mathbf{U}_{0}, \mathbf{U}_{1}, \ldots, \mathbf{U}_{N}\right)^{T}$. For the solution of (6) we can apply the classical Newton's method along with the simple termination criterion

$$
\frac{1}{d(N+1)} \sum_{\ell=1}^{d} \sum_{n=0}^{N}\left|\Delta^{\ell} U_{n}\right| \leq \mathrm{TOL},
$$

where $\Delta^{\ell} U_{n}$, for $n=0,1, \ldots, N$ and $\ell=1,2, \ldots, d$, is the difference between two successive iterate components and TOL is a fixed tolerance.

\section{Numerical Results and Comparison}

In this Section, we present the numerical results obtained by solving the mathematical model (1) using the non-standard finite difference scheme (6) on the quasi-uniform 
grid defined by the logarithmic map (3) with control parameter $c=2$. Now, let us rewrite the model (1) as a first-order system as follows

$$
\begin{aligned}
& \frac{d^{1} u}{d x}={ }^{2} u, \\
& \frac{d^{2} u}{d x}={ }^{3} u, \quad x \in(0, \infty) \\
& \frac{d^{3} u}{d x}=-{ }^{1} u^{3} u-\beta\left(1-{ }^{2} u\right),
\end{aligned}
$$

with

$$
{ }^{1} u(0)={ }^{2} u(0)=0, \quad{ }^{2} u(\infty)=1,
$$

or, in an equivalent form,

$$
\begin{aligned}
& \mathbf{u}=\left({ }^{1} u,{ }^{2} u,{ }^{3} u\right)^{T}, \\
& \mathbf{f}(x, \mathbf{u})=\left({ }^{2} u,{ }^{3} u,-{ }^{1} u^{3} u-\beta\left(1-{ }^{2} u\right)\right)^{T}, \\
& \mathbf{g}(\mathbf{u}(0), \mathbf{u}(\infty))=\left({ }^{1} u(0),{ }^{2} u(0),{ }^{2} u(\infty)-1\right)^{T},
\end{aligned}
$$

where $\mathbf{u}(x)$ is a three-dimensional vector with components ${ }^{\ell} u(x)$ for $\ell=1,2,3$, and $\mathbf{f}:[0, \infty) \times \mathbb{R}^{d} \rightarrow \mathbb{R}^{d}$ and $\mathbf{g}: \mathbb{R}^{d} \times \mathbb{R}^{d} \rightarrow \mathbb{R}^{d}$, with $d=3$.

For all tests we consider the control parameter $c=2$, a fixed tolerance TOL $=10^{-8}$ and, as a first guess for Newton's iteration, the following initial data

$$
{ }^{1} u(x)=0.5 x, \quad{ }^{2} u(x)=1, \quad{ }^{3} u(x)=\exp (-x) .
$$

In Table 1, we report the numerical results obtained for the missing initial data $\frac{d^{2} u}{d x^{2}}$ for increasing points number $N$ and $\beta=1.2$. Here, "iter "stands for the number of the Newton's iterations.

Table 1. Numerical results related to $\frac{d^{2} u}{d x^{2}}(0)$ for different values of $N$.

\begin{tabular}{rll}
\hline$N$ & iter & $\frac{d^{2} u}{d x^{2}}(0)$ \\
\hline 10 & 6 & 1.1790527 \\
20 & 6 & 1.1776846 \\
40 & 6 & 1.1773411 \\
80 & 6 & 1.1772552 \\
160 & 6 & 1.1772336 \\
320 & 7 & 1.1772282 \\
640 & 7 & 1.1772269 \\
1280 & 7 & 1.1772261 \\
\hline
\end{tabular}

In Figure 1, we show the numerical solution obtained for $\beta=1.2$ and $N=80$. The recovered value of the second order derivative of the solution at the origin is $\frac{d^{2} u}{d x^{2}}(0)=1.177255155089504$, obtained in 6 iterations. 


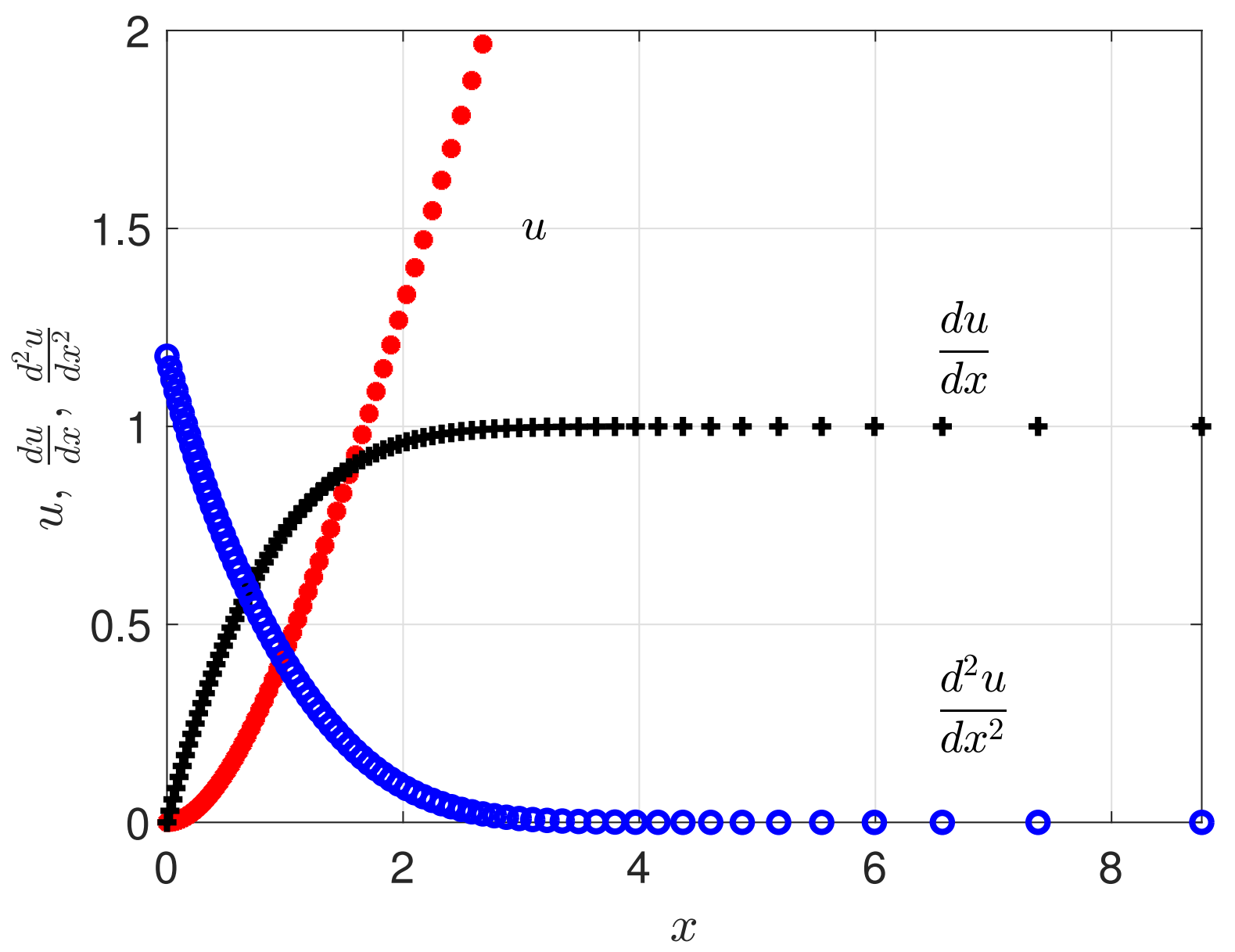

Figure 1. Numerical solution for the problem (1) for $\beta=1.2$ and $N=80$.

The Table 2 lists the obtained numerical results for different values of parameter $\beta$, with $\beta=0,0.2, \cdots, 2$ and $N=100$. For the sake of brevity, we have chosen to report only the values of the wall shear stress, that is the second derivative value at the origin. Within the same table, we can compare our results with those reported by Singh and Chandarki [13]. The problem with $\beta=0$ corresponding to the Blasius problem, and in this case, the computed missing initial condition $\frac{d^{2} u}{d x^{2}}(0)=0.4695839$ can be compared with the value 0.469599988361 computed by Fazio [7] by a free boundary formulation of the Blasius problem.

Table 2. Numerical results, related to $\frac{d^{2} u}{d x^{2}}(0)$, and comparison.

\begin{tabular}{lllc}
\hline$\beta$ & DTM [13] & NIT [13] & FD (this study) \\
\hline 0.0 & 0.46910 & 0.46920 & 0.4695839 \\
0.2 & 0.66343 & 0.64819 & 0.6389912 \\
0.4 & 0.80009 & 0.78749 & 0.7749739 \\
0.6 & 0.91659 & 0.90562 & 0.8917536 \\
0.8 & 1.01988 & 1.01002 & 0.9956343 \\
1.0 & 1.11362 & 1.10460 & 1.0900815 \\
1.2 & 1.20006 & 1.19170 & 1.1772448 \\
1.4 & 1.28068 & 1.27285 & 1.2585668 \\
1.6 & 1.35652 & 1.34913 & 1.3350710 \\
1.8 & 1.42834 & 1.42132 & 1.4075142 \\
2.0 & 1.49671 & 1.49002 & 1.4764751 \\
\hline
\end{tabular}


In order to improve the accuracy of the computed solution, we apply Richardson's extrapolation, using several refinements of the computational domain. On the computational domain of the problem, we build a quasi-uniform grid with a mesh-points number equal to $N_{0}$ and proceed with subsequent grid refinements by constructing meshes with grid-point numbers $N_{g}$ for $g=1,2, \cdots$, where $N_{g+1}=r N_{g}$ with refinement factor $r=2$. On each grid, the numerical solution $U_{g}, g=0,1, \cdots, G$ is computed using the non-standard finite difference method. In order to reduce the calculations, we adopt a continuation strategy, in fact, we use the final solution $U_{g}$ obtained on the grid $g$ as initial guess for calculating the solution $U_{g+1}$ on the grid $g+1$. where the new grid values are approximated by linear interpolations. We define the level of the Richardson's extrapolation by the index $k$ and, the two numerical solutions related to the grids $g$ and $g+1$ at the extrapolated level $k$ by $U_{g, k}$ and $U_{g+1, k}$. We use the following formula to calculate a more accurate approximation

$$
U_{g+1, k+1}=U_{g+1, k}+\frac{U_{g+1, k}-U_{g, k}}{2^{p_{k}}-1} \quad k=0,1, \cdots, G-1 .
$$

In Table 3, we report the extrapolated values with $N=100,200,400$ grid points for $\beta=1$. The last extrapolated value is ${ }^{3} U_{2,2}=1.090064908$ and can be considered as our benchmark value for $\frac{d^{2} u}{d x^{2}}(0)$. We can conclude that the reported extrapolated value is correct up to 9 decimal places.

Table 3. Extrapolated values at origin $x=0$ for $\frac{d^{2} u}{d x^{2}}(0)$ with $\beta=1$.

\begin{tabular}{llll}
\hline$N_{g}$ & ${ }^{3} U_{g, 0}$ & ${ }^{3} U_{g, 1}$ & ${ }^{3} U_{g, 2}$ \\
\hline 100 & 1.090081494 & - & - \\
200 & 1.090069055 & 1.090064908 & - \\
400 & 1.090065945 & 1.090064908 & 1.090064908 \\
\hline
\end{tabular}

\section{Concluding Remarks}

In this paper, the problem (1), that describes the MHD boundary layer flow of an incompressible fluid past a flat plate, is solved by the non-standard finite difference method on a quasi-uniform grid for the different magnetic parameters $\beta$. The values of the second-order derivative of the solution at the origin, for different values of parameter $\beta$, are reported in the Table 2 . In order to verify the accuracy of the proposed method, the results are compared with those by Singh and Chandarki [13]. Moreover, in the case of the Blasius problem, we compare the missing initial condition with the one computed by Fazio [7] by a free boundary formulation of the Blasius problem. The computed values are found to be really accurate.

Data Availability Statement: The data that support the findings of this study are available from the corresponding author upon reasonable request.

Acknowledgments: The research of this work was supported, in part, by the University of Messina and by the GNCS of INDAM.

Conflicts of Interest: The authors declare no conflict of interest.

\section{References}

1. H. Blasius. Grenzschichten in Flüssigkeiten mit kleiner Reibung. Z. Math. Phys., 56:1-37, 1908.

2. F. R. de Hoog and R. Weiss. An approximation theory for boundary value problems on infinite intervals. Computing, 24:227-239, 1980.

3. M. Lentini and H. B. Keller. Boundary value problems on semi-infinite intervals and their numerical solutions. SIAM J. Numer. Anal., 17:577-604, 1980. 
4. P. A. Markowich. A theory for the approximation of solution of boundary value problems on infinite intervals. SIAM J. Math. Anal., 13:484-513, 1982.

5. P. A. Markowich. Analysis of boundary value problems on infinite intervals. SIAM J. Math. Anal., 14:11-37, 1983.

6. G. Kitzhofer, O. Koch, P. Lima, and E. Weinmüller. Efficient numerical solution of the density profile equation in hydrodynamics. J. Sci. Comput., 32:411-424, 2007.

7. R. Fazio. The Blasius problem formulated as a free boundary value problem. Acta Mech., 95:1-7, 1992.

8. J. P. Boyd. Chebyshev and Fourier Spectral Methods. Dover, New York, 2001.

9. J. Shen and L. Wang. Some recent advances on spectral methods for unbounded domains. Commun. Comput. Phys., 5:195-241, 2009.

10. R. Fazio and A. Jannelli. Finite difference schemes on quasi-uniform grids for BVPs on infinite intervals. J. Comput. Appl. Math., 269:14-23, 2014.

11. R. Fazio and A. Jannelli. BVPs on infinite intervals: A test problem, a nonstandard finite difference scheme and a posteriori error estimator. Math. Meth. Appl. Sci., 40:6285-6294, 2017.

12. R. Fazio. Perpetual American put option: an error estimator for a non-standard finite difference scheme. Applied Mathematics E-Notes, 21, 2021. Preprint available at the URL: http:/ / arxiv.org/pdf/1310.4314v1.pdf.

13. B. B. Singh and I. M. Chandarki. Non-integral technique and differential transformation method for MHD boundary layer flow of an incompressible fluid past a flat plate. Int. J. Appl. Math. Res., 1:46-64, 2012.

14. B. Brighi. The equation $\mathrm{f}^{\prime \prime \prime}+\mathrm{ff}^{\prime \prime}+\mathrm{g}\left(\mathrm{f}^{\prime}\right)=0$ and the associated boundary value problems. Results. Math., 61:355-391, 2012.

15. J. D. Lambert. Numerical Methods for Ordinary Differential Systems. Wiley, Chichester, 1991.

16. C. Canuto, M. Y. Hussaini, A. Quarteroni, and Jr. T. A. Zang. Spectral Methods, Fundamentals in Single Domains. Springer-Verlag, Berlin, 2006.

17. A.I. van de Vooren and D. Dijkstra. The Navier-Stokes solution for laminar flow past a semi-infinite flat plate. J. Eng. Math., 4:9-27, 1970. 